\title{
Nonadiabatic scattering of a classical particle in an inhomogeneous magnetic field
}

\author{
F. Evers, A. D. Mirlin, ${ }^{*}$ D. G. Polyakov, ${ }^{\dagger}$ and P. Wölfle \\ Institut für Theorie der Kondensierten Materie, Universität Karlsruhe, 76128 Karlsruhe, Germany
}

(Received 14 July 1998)

\begin{abstract}
We study the violation of the adiabaticity of the electron dynamics in a slowly varying magnetic field. We formulate and solve exactly a nonadiabatic scattering problem. In particular, we consider scattering on a magnetic field inhomogeneity that models scatterers in the composite-fermion theory of the half-filled Landau level. The calculated nonadiabatic shift of the guiding center is exponentially small and exhibits an oscillatory behavior related to the "self-commensurability" of the drifting cyclotron orbit. The analytical results are complemented with a numerical simulation. [S0163-1829(98)08548-8]
\end{abstract}

The dynamics of particles moving in a spatially random magnetic field (RMF) B(r) has been a subject of considerable interest in the past few years. The particular appeal of the subject is due to its relevance to a number of models of strongly interacting disordered electron systems in two dimensions. One of the most prominent examples is the composite-fermion description ${ }^{1}$ of a half-filled Landau level. Within this approach, the electron liquid in a strong magnetic field is mapped-by means of a Chern-Simons gauge transformation-to a fermion gas subject to a weak effective magnetic field. Precisely at half-filling, the expectation value of the Chern-Simons gauge field compensates the effect of the external magnetic field. The RMF appears in this model after taking static disorder into account: fluctuations of the local filling factor due to screening of the random scalar potential yield a local mismatch between the gauge and external magnetic fields. A number of observations ${ }^{2}$ of Fermisurface features near half-filling give strong experimental support to the model of the effective magnetic field. Apart from the composite-particle models involving fictitious fields, two-dimensional (2D) electron systems with a real RMF can be directly realized in semiconductor heterostructures by attaching to the latter superconducting ${ }^{3}$ or ferromagnetic ${ }^{4,5}$ overlayers.

The peculiarity of transport properties of $2 \mathrm{D}$ electrons in a random field $B(\mathbf{r})$ shows up most distinctly in systems with smooth inhomogeneities. The case of long-range disorder is most important also experimentally-since the compressible state in a half-filled Landau level is observed in highmobility samples. In the latter, a large correlation radius of potential fluctuations, $d$, is fixed by a wide "spacer" between the electron gas and the doped layer containing ionized impurities. Likewise, inhomogeneities of the RMF created by the ferromagnetic overlayers in Refs. 4 and 5 appear to be fairly long range. In the composite-fermion model of the half-filled Landau level, the large value of $d$ (as compared to the interelectron distance) allows us to ignore, in the first instance, the quantum interference of scattered waves and describe the electron kinetics as purely classical. Quantum localization effects become crucial either on exponentially long scales or at strong deviations from half-filling, thus motivating us to consider a classical particle subject to the RMF. electron kinetics in the RMF. The conductivity in the field $B(\mathbf{r})=\bar{B}+\delta B(\mathbf{r})$ in the limit of strong homogeneous component $\bar{B}$ was shown in Ref. 6 to be completely determined by weak nonadiabatic processes in scattering on the inhomogeneities $\delta B(\mathbf{r})$ (see also Refs. 7 and 8, where problems were considered). A key ingredient of this transport theory is the nonadiabatic dynamics on the microscopic scale. The purpose of the present paper is to study the microscopic processes of the nonadiabatic scattering in detail by complementing the analytical arguments with results of a numerical simulation.

To this end we formulate a single-scattering problem: we introduce a weak homogeneous gradient of the background magnetic field and consider the interaction of an electron with an "impurity" modeled by a spatially localized perturbation $\delta B(\mathbf{r})$. Far away from the impurity the electron motion is a slow van Alfvén drift along straight lines-which are contours of constant magnetic field-accompanied by rapid cyclotron gyrations. We consider the adiabatic limit, where the shift of the guiding center during one cyclotron period $\delta$, is much smaller than the size $d$ of the impurity, $d / \delta \gg 1$. In this case, the particle continues to drift along the lines of constant field, which is a manifestation of adiabatic invariance. A weak violation of the adiabatic invariance leads to a small shift, after the scattering process has taken place, of the magnetic field contour along which the particle continues its drift. It is this shift, $\Delta \rho$, that will be studied below, both analytically and numerically. The corresponding effect in a system with many impurities governs the magnetoconductivity, as discussed in Ref. 6.

In terms of the complex coordinate $z=x+i y$, the equation of motion at the energy $m v_{F}^{2} / 2$ in our scattering problem reads $\ddot{z}=i \Omega(z) \dot{z}$, where

$$
\Omega(z)=\omega_{c}\left[1+\epsilon \frac{y}{R_{c}}+W(z)\right],
$$

$\omega_{c}=e \bar{B} / m c, \bar{B}$ is the background field at $y=0, R_{c}$ $=v_{F} / \omega_{c}$, the slope $\epsilon$ yields a finite velocity of the incident particle in $x$ direction, and $W(z)$ is a "scattering potential." We assume that $\epsilon \ll 1$. The guiding center coordinate $y$ averaged over the cyclotron orbit, $\rho=\langle y\rangle_{c}$, plays the role of an 
impact parameter. The particle entering the system at $x=$ $-\infty$ with the initial condition $\lim _{x \rightarrow-\infty}\langle y\rangle_{c}=\rho_{i}$ will leave it at $x=\infty$ along the trajectory with $\langle y\rangle_{c}=\rho_{i}+\Delta \rho$, where $\Delta \rho$ is the desired nonadiabatic shift. In order to analyze the scattering problem analytically we assume the impurity field to be weak, $W \ll 1$. Expanding the coordinate $z$ in powers of $W$ : $z=z_{0}+z_{1}+\ldots$, we concentrate on the first-order term $z_{1}$.

The solution $y_{0}$ in the absence of the impurity can be written in an implicit form for the initial conditions $z(0)$ $=0, \quad \dot{z}(0)=i v_{F}$ as $t\left(y_{0}\right)=\omega_{c}^{-1} \int_{0}^{y_{0} / R_{c}} d Y D^{-1 / 2}(Y)$, where $D(Y)=1-Y^{2}[1+(\epsilon / 2) Y]^{2}$. The coordinate $y_{0}$ is confined to the region $y_{-} \leqslant y_{0} \leqslant y_{+}$, where $y_{ \pm} / R_{c}=(\sqrt{1 \pm 2 \epsilon}-1) / \epsilon$, and is periodic with the period $2\left|t_{+}-t_{-}\right| \equiv 2 \pi / \omega$, where $t_{ \pm}=t\left(y_{ \pm}\right)$. The velocity in $x$ direction, $\dot{x}_{0}=-\omega_{c}\left(y_{0}\right.$ $+\epsilon y_{0}^{2} / 2 R_{c}$ ), may be integrated to give $x_{0}=\left\langle\dot{x}_{0}\right\rangle_{c} t+\xi$, where $\xi(t)$ is also periodic with the frequency $\omega$. The leading terms in the small- $\epsilon$ expansion are: $\left\langle\dot{x}_{0}\right\rangle_{c}=\frac{\epsilon}{2} v_{F}$,

$$
\begin{gathered}
\xi=R_{c}\left[(\cos \omega t-1)-\epsilon\left(\sin \omega t+\frac{1}{4} \sin 2 \omega t\right)\right. \\
\left.+O\left(\epsilon^{2}\right)\right], \\
y_{0}=R_{c}\left[\sin \omega t-\frac{3}{4} \epsilon+\epsilon\left(\cos \omega t-\frac{1}{4} \cos 2 \omega t\right)\right. \\
\left.+O\left(\epsilon^{2}\right)\right],
\end{gathered}
$$

and $\omega=\omega_{c}+O\left(\epsilon^{2}\right)$. To find $y_{1}$, we integrate the equation of motion for $x_{1}$ once and use the relation $\dot{x}_{1} \dot{x}_{0}+\dot{y}_{1} \dot{y}_{0}=0$ following from energy conservation, which yields a first-order differential equation for $y_{1}$ with periodic coefficients

$$
\dot{y}_{0} \dot{y}_{1}-\ddot{y}_{0} y_{1}=\omega_{c} \dot{x}_{0} \int_{0}^{t} d t^{\prime} W\left[z_{0}\left(t^{\prime}\right)\right] \dot{y}_{0}\left(t^{\prime}\right) \text {. }
$$

For each half-period between the turning points $t=t_{ \pm}$, at which $\dot{y}_{0}$ changes sign, the solution of Eq. (4) can be represented in the form

$$
y_{1}(t)=\omega_{c} \dot{y}_{0}(t) \int_{0}^{t} d t^{\prime} K\left(t^{\prime}\right) \int_{0}^{t^{\prime}} d t^{\prime \prime} W\left[z_{0}\left(t^{\prime \prime}\right)\right] \dot{y}_{0}\left(t^{\prime \prime}\right) .
$$

Here the factor $K(t)=\dot{x}_{0}(t) / \dot{y}_{0}^{2}(t)$ behaves singularly as $(t$ $\left.-t_{ \pm}\right)^{-2}$ in the vicinity of $t_{ \pm}$. Matching two branches of $y_{1}(t)$ across the turning point requires that the contour of the $t^{\prime}$ integration in Eq. (5) be displaced into the complex plane so as to pass round the singularity on the real axis [since the term $\left(t^{\prime}-t_{ \pm}\right)^{-1}$ is absent in the integrand, the contour can be shifted in either half-plane]. This corresponds to the contraction $\int_{t_{ \pm}}^{t_{ \pm}+\tau} d t\left(t-t_{ \pm}\right)^{-2} \rightarrow-2 \tau^{-1}$ at $\tau \rightarrow 0$. With this choice of the contour of integration, Eq. (5) gives the sought solution at all $t$.

To extract the non-adiabatic shift from Eq. (5), we observe that the integral over $t^{\prime \prime}$ converges to a constant at large $t^{\prime}$ while $\left\langle\dot{y}_{0}\right\rangle_{c}=0$, so that the average $\Delta \rho=\left\langle y_{1}(t \rightarrow \infty)\right\rangle_{c}$ $-\left\langle y_{1}(t \rightarrow-\infty)\right\rangle_{c}$ is given by

$$
\Delta \rho=\alpha I, \quad I=\int_{-\infty}^{\infty} d t W\left[z_{0}(t)\right] \dot{y}_{0}(t),
$$

where $\alpha(\epsilon)=\omega_{c}\left\langle\dot{y}_{0}(t) \int_{0}^{t} d t^{\prime} K\left(t^{\prime}\right)\right\rangle_{c}$ is expressed in terms of the unperturbed solution. At $\epsilon \rightarrow 0$ the constant $\alpha \rightarrow-1$. Note that, apart from the shift $\Delta \rho$, the asymptotics of Eq. (5) at large $t$ contains an oscillating term $-\epsilon I \omega_{c} t \cos \omega_{c} t$, the amplitude of which diverges linearly with growing $t$. This divergency is an artifact of the perturbation expansion in $W$ and reflects the fact that the shift $\Delta \rho$ is accompanied by the change of the frequency $\left(\epsilon \omega_{c} / R_{c}\right) \Delta \rho$. This asymptotics could have been equivalently used to find $\Delta \rho=-I$.

In evaluating $I$ we first assume, for simplicity, that $W(z)$ depends on $x$ only, which allows us to expand $I$ as

$$
I=\int_{-\infty}^{\infty} d t \sum_{n} \frac{1}{n !} \partial_{x}^{n} W\left(\frac{\epsilon}{2} v_{F} t\right) \xi^{n}(t) \dot{y}_{0}(t) .
$$

Since $W\left[(\epsilon / 2) v_{F} t\right]$ is a smooth function of $t$ on the scale of $\omega^{-1}$, the leading contribution to $I$ comes from taking the first harmonic of the integrand-higher harmonics will involve exponentially smaller Fourier components of $W\left[(\epsilon / 2) v_{F} t\right]$. This yields, after integration by parts and resummation,

$$
\begin{gathered}
I \simeq 2 v_{F} \operatorname{Re}\left[A(\epsilon) \int_{-\infty}^{\infty} d t e^{i \omega_{c} t} W\left(\frac{\epsilon}{2} v_{F} t\right)\right], \\
A(\epsilon)=\frac{1}{R_{c}} \int_{0}^{2 \pi / \omega_{c}} \frac{d t}{2 \pi} \exp \left(-\frac{2 i \xi(t)}{\epsilon R_{c}}-i \omega_{c} t\right) \dot{y}_{0}(t) .
\end{gathered}
$$

The integral in Eq. (9) can be evaluated at $\epsilon \ll 1$ by the saddle-point method to give

$$
\Delta \rho=-2 v_{F} \sqrt{\frac{\bar{\epsilon}}{\pi}} \cos \left(\frac{2}{\epsilon}-\frac{\pi}{4}\right) \int_{-\infty}^{\infty} d t \cos \omega_{c} t W\left(\frac{\epsilon}{2} v_{F} t-R_{c}\right) .
$$

This equation is a parametrically exact solution of the scattering problem at $\epsilon \rightarrow 0$. It expresses the nonadiabatic shift in terms of the asymptotics of the Fourier transform of the smooth function $W$-thus demonstrating explicitly the exponential smallness of $\Delta \rho$. The parameter that governs the exponential falloff of $\Delta \rho$ is $d / \delta \gg 1$, where $d$ is a characteristic scale of variation of $W$ and $\delta=\pi \epsilon R_{c}$ is the shift of the guiding center after one period of the cyclotron rotation, while the ratio $d / R_{c}$ may be arbitrary. Though the limit of a smooth inhomogeneity on the scale of $R_{c}$ is historically most closely associated with the notion of adiabaticity, the parameter $d / R_{c}$ plays no role in Eq. (10). It is worth noting that the preexponential factor given by the first line of Eq. (10) is a nonanalytic function of the small parameter $\epsilon$ and, moreover, happens to oscillate wildly as $\epsilon \longrightarrow 0$. These oscillations are due to the commensurability of two length scales $R_{c}$ and $\delta$. Remarkably, the series of the geometric resonances that constitute the oscillations is associated with the properties of the unperturbed solution ("self-commensurability") and not with the shape of the scatterer. Note that to get the oscillations one has to sum up all terms in the expansion (7) even if $W$ is smooth on the scale of $R_{c}$. Another peculiar feature of the nonadiabatic shift is related to its sensitivity to the phase $\phi$ of the cyclotron rotation of the incident electron. Specifically, changing the position of the scatterer by $\Delta x$ leads to the oscillations of $\Delta \rho(\phi)=\Delta \rho_{m} \cos \left(\phi-\phi_{0}\right) \propto \int_{-\infty}^{\infty} d t \cos \left(\omega_{c} t\right.$ 


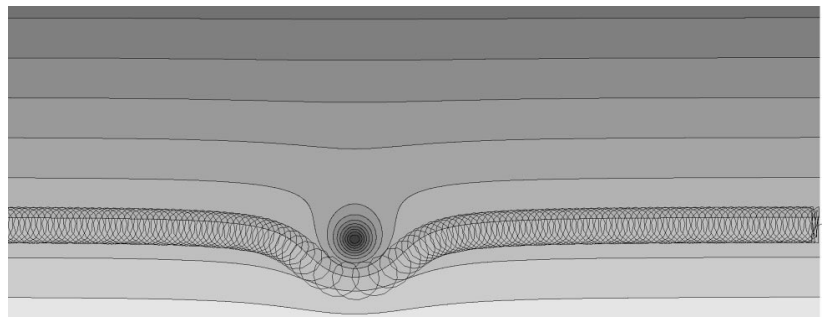

FIG. 1. Typical trajectory of a particle scattered on a "magnetic impurity" [Eq. (1)]. The lines of constant magnetic field $B(\mathbf{r})$ are shown. The strength of the impurity $W_{0}=2.2$.

$+\phi) W\left[(\epsilon / 2) v_{F} t\right]$, where $\phi=2 \pi \Delta x / \delta$, so that the shift vanishes periodically with varying initial conditions.

In the composite-fermion problem, a charged impurity located the distance $d$ from the plane occupied by the electron gas creates the axially symmetric perturbation $W(\mathbf{r})$ $=W_{0} d^{3}\left[(\mathbf{r}-\mathbf{R})^{2}+d^{2}\right]^{-3 / 2}$. We choose the impurity position $\mathbf{R}=\left(0, \rho_{i}\right)$, so that $\rho_{i}$ has the meaning of the impact parameter with which the guiding center is incident on the impurity. Extracting the first-order Fourier component of $W\left[z_{0}(t)\right]$ in the same way as in Eqs. (8),(9), we get

$$
\begin{aligned}
I \simeq & 2 \operatorname{Re} \int_{0}^{2 \pi / \omega_{c}} \frac{d t}{2 \pi} e^{-2 i \xi(t) / \epsilon R_{c}-i \omega_{c} t} \dot{y}_{0}(t) \omega_{c} \\
& \times \int_{-\infty}^{\infty} d t^{\prime} e^{i \omega_{c} t^{\prime}} W\left[\frac{\epsilon}{2} v_{F} t^{\prime}, y_{0}(t)\right] .
\end{aligned}
$$

The function $W$ in this equation has branch points at $t^{\prime}$ $=t_{s}^{\prime}(t)$, where $t_{s}^{\prime}(t)= \pm\left(2 i / \epsilon v_{F}\right) \sqrt{d^{2}+\left[y_{0}(t)-\rho_{i}\right]^{2}}$, which determines the exponentially small value of the integral over $t^{\prime}$. Specifically, the second line in Eq. (11) reads $\sqrt{2 \pi} W_{0}(2 \pi d / \delta)^{3}\left[\omega_{c}\left|t_{s}^{\prime}(t)\right|\right]^{-3 / 2} \exp \left[-\omega_{c}\left|t_{s}^{\prime}(t)\right|\right]$. The remaining integral over $t$ can be done by the saddle-point method. The cumbersome general expression reduces to

$$
\begin{aligned}
\Delta \rho \simeq & 8 \pi W_{0}\left(\rho_{i}+\widetilde{d}\right)\left(\frac{R_{c} d}{\delta^{2}}\right)^{1 / 2} e^{-\rho_{i} / \widetilde{d}} \\
& \times \cos \phi \cos \left(\frac{2 d}{\epsilon \widetilde{d}}-\frac{\pi}{4}\right) \exp \left(-2 \pi \frac{\widetilde{d}}{\delta}\right)
\end{aligned}
$$

in the limit of a long-range impurity potential, when $d,\left|\rho_{i}\right|$ $\gg R_{c}$. Here $\widetilde{d}=\left(d^{2}+\rho_{i}^{2}\right)^{1 / 2}$. In the opposite case, when $R_{c}$ is larger than both $d$ and $\left|\rho_{i}\right|$, we get

$$
\Delta \rho \simeq 8 \pi W_{0} \frac{d^{2}}{\delta} \cos \phi \cos \frac{2}{\epsilon} \exp \left(-2 \pi \frac{d}{\delta}\right) .
$$

The last three factors in Eqs. (12),(13) reflect the features of the nonadiabatic shift discussed above: the exponential smallness, the oscillations with changing $\epsilon$, and the oscillatory dependence on the phase $\phi$. Note that all the formulas above imply that the drift trajectory is only slightly perturbed by $W(\mathbf{r})$.

Now let us turn to a numerical simulation. A typical trajectory resulting from the numerical integration of Eq. (3) is shown in Fig. 1. Though the perturbation of the drift trajectory is seen to be large in this particular example, the nonadibatic shift is almost invisible. The inset to Fig. 2 shows

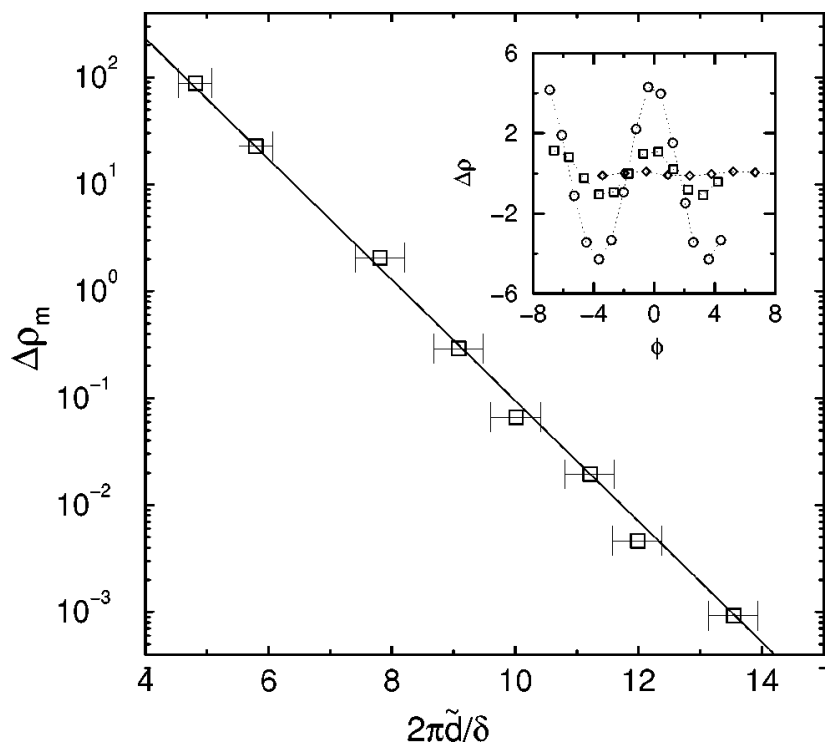

FIG. 2. Amplitude of the nonadiabatic shift $\Delta \rho_{m}$ as a function of $\delta=2 \pi v_{d} / \omega$ for a strong impurity: $W_{0}=2.2$. The solid line is a fit $\Delta \rho_{m} \propto \exp [-1.3 \times 2 \pi \widetilde{d} / \delta]$. Inset: Shift $\Delta \rho$ as a function of the phase $\phi$ for different values of the parameter $2 \pi \widetilde{d} / \delta$ $=4.8(\bigcirc), 5.8(\square), 7.8(\diamond)$.

the periodic dependence of the shift on the phase $\phi$, which agrees with Eq. (12). The main panel clearly demonstrates the adiabatic character of the scattering at $\widetilde{d} / \delta \gg 1$ : the magnitude of the oscillations $\Delta \rho_{m}$ plotted against $\delta^{-1}$ is seen to fall off exponentially, also as predicted by Eq. (12). The slope $c$ of the exponential decay $\ln \Delta \rho=-2 \pi c \widetilde{d} / \delta$ is found to be 1.3 , which is somewhat larger than the value $c=1$ following from Eq. (12). This is because the perturbation of the drift trajectory is not negligible in this case. No oscillations with changing $\epsilon$ could be reliably seen in Fig. 2, which should also be ascribed to their smearing due to the curvature

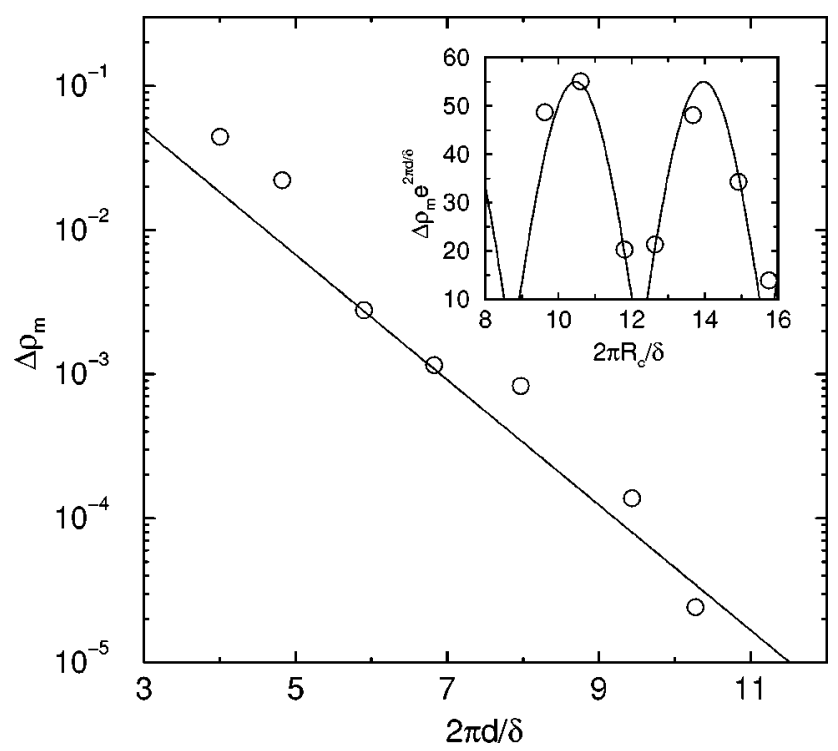

FIG. 3. Nonadiabatic shift for a weak impurity $\left(W_{0}=0.07\right)$ at $\rho_{i} / d=0.4-0.9$ and large $R_{c} / d=1.5-2.5$. The solid line is a fit $\Delta \rho_{m} \propto \exp (-2 \pi d / \delta)$. Inset: Oscillations of $\Delta \rho_{m}$ as a function of $R_{c} / \delta$. 
of the trajectory. Indeed, the commensurability of $R_{c}$ and $\delta$ cannot be maintained in the whole region of interaction if the drift trajectory is strongly perturbed, as clearly illustrated by Fig. 1. The nonadiabatic shift at a smaller amplitude of the interaction $W_{0}$ is shown in Fig. 3. In this case, the perturbation of the trajectory was weak, and the particle was essentially drifting right "through the impurity" with a small impact parameter $\rho_{i}<d, R_{c}$. Decreasing $W_{0}$ narrows the range of the numerical simulation but clearly reveals the oscillatory behavior of $\Delta \rho_{m}$ with changing $\epsilon$. The exponential dependence in Fig. 3 is fitted very well by $e^{-2 \pi d / \delta}$ [in accordance with Eq. (13)] with no fitting parameter in the exponent. The oscillations of $\Delta \rho_{m}$ with changing $R_{c} / \delta$ (inset of Fig. 3) have a period close to $1 / 2$ and are in good agreement with the analytical prediction $\Delta \rho_{m} \propto\left|\cos \left(2 \pi R_{c} / \delta\right)\right|$.

In conclusion, we have studied the violation of the adiabaticity of the electron dynamics in a slowly varying magnetic field. We formulated a scattering problem which has been solved exactly. As a particular example we considered scattering on a single "magnetic impurity" which models a scatterer in the composite-fermion theory of the half-filled Landau level. The nonadiabatic shift of the guiding center is exponentially small and exhibits oscillations with $R_{c} / \delta$, where $R_{c}$ is the cyclotron radius, and $\delta$ the shift of the guiding center along the drift trajectory after one cyclotron period. The oscillations are related to the selfcommensurability of the drifting cyclotron orbit. The analytical results are in full agreement with the numerical simulation. A detailed numerical study of the transport properties of the fermions in a random magnetic field will be presented elsewhere.

This work was supported by the Deutsche Forschungsgemeinschaft through SFB 195 and by the Graduiertenkolleg "Kollektive Phänomene im Festkörper,' and by the INTAS through Project No. 97-1342.
*Also at St. Petersburg Nuclear Physics Institute, 188350 St. Petersburg, Russia.

${ }^{\dagger}$ Also at A.F. Ioffe Physico-Technical Institute, 194021 St. Petersburg, Russia.

${ }^{1}$ B. I. Halperin, P. A. Lee, and N. Read, Phys. Rev. B 47, 7312 (1993); N. Read (unpublished).

${ }^{2}$ R. L. Willett, Adv. Phys. 46, 447 (1997) and references therein.

${ }^{3}$ A. Geim, S. Bending, and I. Grigorieva, Phys. Rev. Lett. 69, 2252 (1992).

${ }^{4}$ P. D. Ye, D. Weiss, G. Lütjering, R. R. Gerhardts, K. von Klitzing, K. Eberl, H. Nickel, and G. Weimann, in Proceedings of the 23rd International Conference on The Physics of Semiconductors (World Scientific, Singapore, 1996), p. 1529.

${ }^{5}$ L. Zielinski, K. Chaltikian, K. Birnbaum, C. M. Marcus, K. Campman, and A. C. Gossard, Europhys. Lett. 42, 73 (1998).

${ }^{6}$ A. D. Mirlin, D. G. Polyakov, and P. Wölfle, Phys. Rev. Lett. 80, 2429 (1998).

${ }^{7}$ M. M. Fogler, A. Yu. Dobin, V. I. Perel, and B. I. Shklovskii, Phys. Rev. B 56, 6823 (1997).

${ }^{8}$ G. M. Zaslavsky, R. Z. Sagdeev, D. A. Usikov, and A. A. Chernikov, Weak Chaos and Quasi Regular Patterns (Cambridge University, Cambridge, 1991). 